\title{
Aplicación de un módulo autoinstructivo de matemática y su efecto sobre el rendimiento académico en estudiantes universitarios
}

Application of a self-instructional module of mathematics and its effect on academic performance in university students

Jhonny Javier Albitres Infantes ${ }^{1}$, Lucy Estela Salinas Flores ${ }^{2}$

\section{RESUMEN}

Objetivo: Determinar la influencia de la aplicación de un módulo autoinstructivo de matemática en el rendimiento académico de los estudiantes Universitarios de Ingeniería de sistemas de la Universidad Nacional José Faustino Sánchez Carrión. Métodos: Se realizó una investigación cuasiexperimental con dos grupos, de control y un grupo experimental con pre y post test. La muestra estuvo conformada por 47 estudiantes de los cuales 23 eran del grupo experimental y 24 del grupo de control, a quienes se le aplicó una prueba de entrada y una prueba de salida, constituida por tres tipos de evaluación que median los aspectos: razonamiento y demostración, comunicación matemática y resolución de problemas, durante un periodo de cuatro meses. Para la contrastación de las hipótesis se utilizó la prueba de "t" de Student. Resultados: Los resultados muestran que la aplicación del módulo autoinstructivo de matemática tuvo un efecto significativo sobre el razonamiento y demostración, comunicación matemática y en la resolución de problemas, mejorando el rendimiento en los estudiantes universitarios. Conclusión: La aplicación de módulos autoinstructivos es la mejor alternativa para elevar el rendimiento académico.

Palabras clave: Módulo autoinstructivo, rendimiento académico, razonamiento y demostración, comunicación matemática y resolución de problemas.

\section{ABSTRACT}

Objective: Determine the influence of the application of a self-instructive module of mathematic on the academic performance of the university students of systems Engineering of the national university "José Faustino Sánchez Carrión". Methods: A quasi-experimental investigation was carried out with two control groups and one experimental group with pre and post test. The sample consisted of 47 students of which 23 were from the experimental group and 24 from the control group, to whom an entrance test and an exit test were applied, constituted by three types of evaluation that mediate the aspects: reasoning and demonstration, mathematical communication and problem solving, during a period of four months. For the verification of the hypothesis the test of "t" of Student, was used. Results: The results show that the application of the self-instructive module of mathematic had a significant effect about the reasoning and demonstration, mathematical communication and problem solving, improvind the performance in the university students. Conclusion: The application of self-instructive modules is the best alternative to raise the academic performance.

Keywords: self-instructive module, Academic Performance, reasoning and demonstration, mathematical communication and problem solving.

\footnotetext{
${ }^{1}$ Facultad de Ciencias. Universidad Nacional José Faustino Sánchez Carrión. Huacho, Perú.

${ }^{2}$ Facultad de Ciencias Empresariales. Universidad Nacional José Faustino Sánchez Carrión. Huacho, Perú.
} 


\section{INTRODUCCIÓN}

En estos últimos años, grandes cambios han generado la existencia de una dinámica social rápida, profunda en contenido y global en extensión, estos cambios acelerados que la sociedad enfrenta en las últimas décadas, ha dado lugar a que se le denomine la era del conocimiento y la información; porque para alcanzar y mantener adecuados niveles de desarrollo económico y social, se requiere acceder a la información, materia prima del conocimiento, que a su vez constituye la riqueza más importante de un país (Niño, 2011).

Por consiguiente, los jóvenes de hoy necesitan aprender matemáticas. Los desafíos a los que se enfrenta la sociedad contemporánea han provocado la prolongación progresiva del nivel educativo. $Y$ en esta educación el papel de las ciencias en general en especial las matemáticas no han hecho otra cosa que crecer. No basta con saber leer, escribir y hacer cuentas, es necesario poderse expresar oralmente y por escrito sobre temas complejos y poder discutir sobre ellos; hay que dominar también técnicas sofisticadas, para las que se exigen conocimientos matemáticos referidos a las grandes estructuras de la aritmética, del álgebra, del análisis y de la geometría, técnicas que hace un siglo estaban limitadas a un círculo restringido (Fernández, 2000).

Por otro lado cambios sorprendentes vienen sucediendo en el ámbito educativo, y nos exige centrar nuestra atención en los elementos esenciales, como es el aprendizaje y los métodos de enseñanza, por ello, consideramos que el proceso de enseñanza aprendizaje, requiere ser innovado especialmente en el sistema educativo de nivel superior, ya que aún se sigue impartiendo conocimientos en muchos centros de este nivel, con métodos tradicionales.

Según Miranda (2011) indica que en una sociedad donde los avances científicos y tecnológicos van cambiando e influyendo vertiginosamente de manera determinante en todas las tareas educativas, es imprescindible que el docente los adopte para formar estudiantes competentes en el que sean constructores de sus propios aprendizajes.

No hay duda de la importancia de la Matemática en la formación universitaria, pero según registros académicos muestran que, el rendimiento académico en matemáticas de nuestros estudiantes universitarios no es bueno y en especial en los estudiantes de la Escuela Profesional Ingeniería de Sistemas, situación que preocupa a los docentes de la especialidad.

El análisis de esta realidad ha motivado la necesidad de trabajar la presente investigación, con la aplicación de los módulos autoinstructivos. Los módulos autoinstructivos se definen como materiales diseñados para permitir el logro de los objetivos educativos, prescindiendo del trabajo del docente en la mayor parte del aprendizaje sin llegar a suplirlo (Loayza, 1999). Para Negret (2001) el módulo autoinstructivo, es un material escrito impreso, a veces llamado guía didáctica o texto guía; es un material que igualmente se puede presentar en forma virtual; facilita la búsqueda y acceso rápido de la información; no ofrece sólo contenidos sino también, recomendaciones, actividades, evaluaciones, notas, consideraciones especiales, etc.

Por consiguiente, el módulo autoinstructivo en matemática II elaborada sistemáticamente por el docente, está formada por un conjunto de actividades organizadas y diversificadas en torno al conocimiento de la asignatura, que tenga a su vez, un carácter autodidáctico y autosuficiente, para elevar el nivel de las capacidades de matemática II y como ende elevar el nivel del rendimiento académico de los estudiantes. Por tanto, la presente investigación consiste básicamente en determinar la influencia de la aplicación de un módulo autointructivo de matemática II en el rendimiento académico de los estudiantes de la escuela profesional de Ingeniería de Sistemas de la Universidad Nacional José Faustino Sánchez Carrión.

\section{MATERIAL Y MÉTODOS}

Se ha utilizado una muestra no-probabilistica por 47 estudiantes, 23 estudiantes para el grupo experimental y 24 estudiantes para el grupo de control, cuya población es de 56 estudiantes. Los instrumentos utilizados 
fueron dos pruebas (Pre-test y Pos-test) de 30 ítems cada una para evaluar las capacidades en matemáticas II teniendo en cuenta las capacidades de razonamiento y demostración (10 Ítems), comunicación matemática (10 ítems) y resolución de problemas (10 ítems), los 47 estudiantes rindieron el pre-test. Se aplicó el programa "Modulo autoinstructivo en matemática II" al grupo experimental, y al grupo de control se aplicó el método tradicional, al final del programa se aplicó el pos-test a las 47 estudiantes.

La igualdad de variancia fue evaluada con la prueba de Levene, y se aplicó la prueba t-

\section{RESULTADOS}

Tabla 1. Nivel Rendimiento Académico de los estudiantes en la etapa Pre - Test

\begin{tabular}{|c|c|c|c|c|}
\hline \multirow[t]{2}{*}{ Nivel } & \multicolumn{2}{|c|}{$\begin{array}{l}\text { Control } \\
\text { Método tradicional }\end{array}$} & \multicolumn{2}{|c|}{$\begin{array}{l}\text { Experimental } \\
\text { Módulo Autoinstructivo }\end{array}$} \\
\hline & Frecuencia & $\%$ & Frecuencia & $\%$ \\
\hline Muy malo & 4 & 16,7 & 3 & 13 \\
\hline Malo & 20 & 83,3 & 20 & 87 \\
\hline Regular & 0 & 0 & 0 & 0 \\
\hline Bueno & 0 & 0 & 0 & 0 \\
\hline Muy bueno & 0 & 0 & 0 & 0 \\
\hline Total & 24 & 100 & 23 & 100 \\
\hline
\end{tabular}

La Tabla 1 muestra el nivel académico de los estudiantes en la etapa pre - test. El rendimiento académico en los estudiantes del grupo de control es muy malo con un $16,7 \%$ y malo con un $83,3 \%$, mientras el rendimiento académico en los estudiantes del grupo experimental fue muy malo con un $13 \%$ y malo con un $87 \%$.

Tabla 2. Nivel de aceptación del módulo autoinstructivo en matemática Il de los estudiantes del II ciclo de la EAP de Ingeniería de Sistemas

\begin{tabular}{lcc}
\hline Nivel & Frecuencia & Porcentaje \\
\hline Regular & 2 & \\
Bueno & 8 & 8,7 \\
Muy bueno & 13 & 34,8 \\
& & 56,5 \\
\hline Total & 23 & 100 \\
\hline
\end{tabular}

El nivel de aceptación del módulo autoinstructivo en matemática II de los estudiantes del grupo experimental es regular con un $8,7 \%$, bueno con un $34,8 \%$ y muy bueno con un $56,5 \%$; tal como lo observamos en la Tabla 2.

Tabla 3. Efecto de la aplicación del módulo autoinstructivo de matemática II sobre el rendimiento académico de los estudiantes.

\begin{tabular}{lcc} 
Capacidad & $\begin{array}{c}\text { Control } \\
\text { Método Tradicional } \\
\text { Media } \pm \text { desviación } \\
\text { estándar }\end{array}$ & $\begin{array}{c}\text { Experimental } \\
\text { Módulo autoinstructivo } \\
\text { Media } \pm \text { desviación } \\
\text { estándar }\end{array}$ \\
\hline $\begin{array}{l}\text { Razonamiento y } \\
\text { demostración }\end{array}$ & $8,25 \pm 2,38^{\mathrm{b}}$ & $13,22 \pm 1,98^{\mathrm{a}}$ \\
$\begin{array}{l}\text { Comunicación } \\
\text { matemática }\end{array}$ & $9,75 \pm 2,52^{\mathrm{b}}$ & $12,35 \pm 2,23^{\mathrm{a}}$ \\
$\begin{array}{l}\text { Resolución de } \\
\text { problemas } \\
\text { General }\end{array}$ & $9,67 \pm 2,33^{\mathrm{b}}$ & $12,43 \pm 1,80^{\mathrm{a}}$ \\
\hline
\end{tabular}


La prueba de Levene encontró normalidad entre los datos evaluados ( $p>0,05)$, por lo que seguidamente realizamos la comparación de las medias de las variables evaluadas. La prueba $t$ Student encontró diferencias estadísticas significativas de las capacidades, entre los método evaluados $(p<0,05)$. Las capacidades de razonamiento y demostración, comunicación matemática, y resolución de problemas fueron superiores en los alumnos que recibieron el módulo autoinstructivo comparado con el método tradicional. (Tabla 3). Estos resultados sugieren que al $95 \%$ de confianza se puede afirmar que existe evidencias estadística para concluir que la aplicación de un módulo autoinstructivo de matemática II si influye significativamente en la capacidad de resolución de problemas de los estudiantes universitarios de Ingeniería de sistemas.

Los resultados alcanzados en la evaluación de entrada al grupo experimental nos proporciona un promedio de 6,96 puntos en la escala vigesimal, mientras que el promedio del grupo de control es de 6,58 puntos; estos resultados nos permite concluir que entre ambos grupos no existe diferencias significativas en sus niveles de aprendizaje, demostrándonos que ambos grupos poseen características homogéneas e inician el proceso de investigación en similares condiciones.

Los resultados obtenidos luego de la evaluación de salida al grupo experimental que empleo módulo autoinstructivo en sus aprendizajes, nos proporcionan un promedio de 12,4 puntos en escala vigesimal que fue superior al promedio alcanzado por el grupo de control de 9,25 puntos, grupo que empleo en su aprendizaje el método tradicional.

Los resultados para la capacidad de razonamiento y demostración de los alumnos del módulo autoinstructivo nos proporcionan un promedio de 13,22 puntos superior al promedio alcanzado por el grupo de control de 8,5 puntos, grupo que empleo en su aprendizaje el método tradicional.

Los resultados obtenidos para la capacidad comunicación matemática de los alumnos del módulo autoinstructivo, nos proporcionan un promedio de 12,35 puntos superior al promedio alcanzado por el grupo de control de 9,75 puntos, grupo que empleo en su aprendizaje el método tradicional.

Los resultados obtenidos para la capacidad resolución de problemas de los alumnos del módulo autoinstructivo, nos proporcionan un promedio de 12,43 puntos en escala vigesimal, respecto al promedio alcanzado por el grupo de control de 9,67 puntos, grupo que empleo en su aprendizaje el método tradicional; lo que significa que la aplicación de un módulo autoinstructivo de matemática II si influye significativamente en el rendimiento de la capacidad de resolución de problemas de los estudiantes universitarios de la Escuela Profesional de Ingeniería de sistemas.

\section{DISCUSIÓN}

Cámac (2018) en su trabajo de investigación titulada "Los textos autoinstructivos en el aprendizaje significativo en estudiantes de educación primaria de la Universidad Nacional de Educación Enrique Guzmán y Valle", concluye que existe una alta relación entre el texto autoinstructivo y el aprendizaje significativo de proposiciones en estudiantes de educación primaria. Asimismo, Niño (2011) al evaluar la aplicación de módulos autoinstructivos en la especialidad de construcciones metálicas con respecto del método tradicional encontró que los alumnos alcanzan aprendizajes significativos. Huaraca (2012) en su investigación titulada "Módulo instructivo y el aprendizaje significativo de matemática en estudiantes de nivel secundaria de la institución educativa de Quinua", concluye que con la aplicación del módulo instructivo la mayoría de los estudiantes lograron los aprendizajes previstos entre excelente y bueno, porque han desarrollado satisfactoriamente las capacidades de razonamiento y demostración, comunicación matemática y resolución de problemas.

Estos resultados coinciden con los de la presente investigación, donde la aplicación del módulo autoinstructivo de matemática II influyó significativamente en el rendimiento académico de los estudiantes de Ingeniería de sistemas. Por tanto la aplicación de módulos autoinstructivos es la mejor alternativa para elevar el rendimiento académico. 


\section{REFERENCIAS BIBLIOGRÁFICAS}

Cámac, S. (2018). Los textos autoinstructivos en el aprendizaje significativo en estudiantes de educación primaria de la Universidad Nacional de Educación Enrique Guzmán y Valle, 2015. Tesis para optar el grado de doctor en educación. Universidad José Carlos Mariátegui, Moquegua, Perú.

Huaraca, Y. (2012). Módulo instructivo y el aprendizaje significativo de matemática en estudiantes de nivel secundaria de la institución educativa de Quinua, 2010. Tesis para optar el grado de maestro en educación. Universidad Nacional San Cristóbal de Huamanga, Ayacucho.

Loayza, G. (1999) Material Educativo. Madrid. Ediciones Bruner.

Miranda, V. (2011). Módulos Autoinstructivos para el logro de competencias en Estadística Descriptiva de los estudiantes del VI ciclo del Instituto Superior Pedagógico Público José Jiménez Borja de la Ciudad de Tacna. Universidad Nacional Jorge Basadre Grohmann. Tacna: Perú.
Niño, O. (2011). La aplicación de módulos autoinstructivos y su eficacia en el aprendizaje de la soldadura por arco eléctrico de los estudiantes de la Universidad Nacional de Educación Enrique Guzmán y Valle. Lima.

Negret, M. (2001). La selección y el diseño de medios de comunicación didácticos para el proceso de enseñanza aprendizaje. Santa Fe, Colombia

Fernández, G. (2000). Pedagogía, psicología y didáctica de la matemática.

Obtenido de

http://150.214.55.100/asepuma/sevila 2000/m3-01.pdf

Correo electrónico: jjai1426@hotmail.com

Revisión de pares:

Recibido: 15-04-2019

Aceptado: 21-06-2019 